\title{
Incidence of Smear-positive Tuberculosis in Dale District, Sidama, South Ethiopia
}

\author{
Endrias Markos Woldesemayat \\ School of Public Health, Hawassa University, Hawassa, Ethiopia
}

Email address:

endromark@yahoo.com

To cite this article:

Endrias Markos Woldesemayat. Incidence of Smear-positive Tuberculosis in Dale District, Sidama, South Ethiopia. American Journal of Clinical and Experimental Medicine. Vol. 9, No. 2, 2021, pp. 22-27. doi: 10.11648/j.ajcem.20210902.11

Received: January 28, 2021; Accepted: February 26, 2021; Published: April 1, 2021

\begin{abstract}
Background: Estimating TB incidence rate using follow-up study has not been done in southern Ethiopia. Objective: The aim of this study was to estimate incidence rate of smear-positive TB in Dale district, Sidama Region, South Ethiopia. Methods: In a cohort study conducted among 36,575 people in six rural communities: 14,801 children, 92 cases with history of TB treatment and 15 smear-positive TB cases were excluded from the cohort. For 648 smear-negative patients with symptoms of TB and 1443 neighbourhood controls, we did a 3 - 4 monthly follow-up surveys and detected TB cases. For the rest of population, at the end of the follow-up, identification of TB cases from the district TB register was done. Results: In this study, 21,667 adult people were followed for 18,031 person-years and detected 49 new TB cases. Incidence rate of smear-positive TB was $(273 ; 95 \%$ CI: $206-361) / 100,000$ person-years. The risk of smear-positive TB was higher among illiterate people (Adjusted incidence rate ratio 2.4; $95 \%$ CI $1.2-5.0$ ). Conclusion: TB continued to be a major public health problem in rural communities of South Ethiopia. A high incidence rate of smear-positive TB was observed in the study settings. To improve specificity of the diagnosis, future studies should consider using sputum culture or GeneXpert in screening and diagnosis of TB.
\end{abstract}

Keywords: Follow-up, Incidence Rate, Ethiopia, TB

\section{Introduction}

The overall goal of the strategy is to end the global TB epidemic, with corresponding 2035 targets of a $95 \%$ reduction in TB deaths, a 90\% reduction in TB incidence (both compared with 2015) and a target of zero catastrophic costs for TB affected families by 2035 [1]. Therefore, it is important to have a reliable measurement of TB incidence. Direct measurement of TB incidence is the most reliable method [2]. Since TB is a rare disease, determining its incidence through direct measurement requires long-term prospective population based large cohort studies. This makes TB incidence study expensive [3, 4].

In southern Ethiopia, a community randomized trial among adults in Hadiya zone reported smear-positive TB notification rate of 207/100,000 in 2004 [5]. Smear-positive TB notification rate among the whole population in Sidama zone was $127 / 100,000$ population in 2011 [6]. The strategy included advocacy, training, engaging stakeholders (communities) and TB case-finding by HEWs. Community health promoters (CHP) conducted house-to-house visits, identified and informed symptomatic individuals how to produce sputum and did repeated visit to smear-negative cases [6]. These studies indicated that magnitude of the problem in the South Ethiopia region is high $[5,6]$.

In 2011, a study from Dabat district in northern Ethiopia, which used a TB surveillance system among 46,165 residents older than 14 years of age, reported an incidence rate of smear-positive TB 311/100,000 person-years [7]. The corresponding rate during 2009 in rural China among all age groups was 36.7/100,000 person-year in Xiangtan, and 22.7/100,000 person-year in Danyang [8]. In this study, the TB cases were detected through routine detection, strengthening of high-risk groups screening and household contact investigations and they used a 3 times sputum microscopy and sputum culture [8].

Many other reports on incidence of TB are based on the national TB notification data, which may give reliable estimate for developed countries [9]. However, using such reports may not be appropriate for countries like Ethiopia. Delay in diagnosis, caused by low health service coverage and limited TB diagnostic facilities, could contribute to low 
case-finding of TB which results in faulty $\mathrm{TB}$ incidence estimate [10-12]. A recent report showed that there are areas in Sidama inaccessible to TB diagnostic facilities [13]. TB incidence reports in such areas often lack accuracy and the true burden is unknown [14]. Therefore, the Ministry Of Health (MOH) of Ethiopia gave due attention on strengthening TB case detection and management at community level [15].

In 2003, Ethiopia adopted a community-based health extension programme (HEP) [16]. Two women from each kebele, who completed high school, receive one-year training and they are placed as HEWs in their respective kebeles, the smallest administrative unit [17]. The HEWs provide health education, identify and refer presumptive $\mathrm{TB}$ cases, trace defaulters and ensure treatment adherence.

In 2011, the national case-detection rate (CDR) of smearpositive TB was $36.8 \%$ [18]. The corresponding rate for southern Ethiopia region was $48.4 \%$ [18]. Lack of populationbased baseline data and assumption of high HIV prevalence $(6 \%)$ overestimated the TB burden as a result, low case detection rate was reported $[18,19]$. In reality, prevalence of HIV was only $2.4 \%$ (0.9\% in rural areas) for Ethiopia [20]. After the national TB prevalence survey report, the revised 2011 WHO CDR of smear-positive TB for the country was around $72 \%$ [20]. Similar measure for the southern Ethiopia region could be higher than the previous estimate. However, the rates are still low and the true incidence rate of smearpositive TB may be underestimated. Moreover, estimating TB incidence rate using follow up study has not been done in southern Ethiopia region. This study aimed to estimate incidence rate and determine risk factors of new smearpositive TB among adults in Dale district, South Ethiopia.

\section{Methods}

\subsection{Study Site}

During the field work of this study, Sidama Zone in South Ethiopia had 19 districts and 2 urban administrations, which was further subdivided into 524 rural and 39 urban kebeles. Each kebele had at least one government health post. Implementation of the directly observed treatment short course (DOTS) strategy was started in 1995 [21]. The TB control service coverage in the Zone had increased from $37 \%$ in 2003 to $73 \%$ in 2012 [13].

The study was done in Dale district, which was selected purposely because of its high TB burden and accessibility to conduct the study. Dale district is a densely populated agrarian community with about 250,000 people. Out of 36 kebeles in the district, the study was carriedout in randomly selected six kebeles. All eligible people in the selected kebeles were involved in the study.

\subsection{Study Design and Population}

First, a census was done from August 29 - September 10, 2011, which involved TB symptom screening and sputum microscopy. Using a structured and pre-tested questionnaire, any adult person living in each household was interviewed and socio-demographic information was collected for all members of the household. Interviewees were asked whether or not any member of the household was currently experiencing a cough for two weeks or more. Then patients were asked if they have other TB suggestive symptoms such as blood in the sputum, fever, loss of appetite, weight loss, chest pain, shortness of breath, and night sweats. Sputum smear investigation was done as required. A total of 36,575 people, who lived in 7,212 households were enumerated, of whom 14,801 were children, 92 had history of treatment for TB and 15 people were diagnosed with smear-positive TB by the census, so were excluded from the study.

The census identified smear-negative patients with symptoms of $\mathrm{TB}$ and neighbourhood controls. Then we followed them for ten months (from September 2011 to June 2012) and detected TB cases [22]. For the rest of population living in the study communities, at the end of the follow-up, we identified TB cases from the district registries of the National Tuberculosis Control Program.

Smear-negative patients with symptoms of TB were 648 people with cough of two weeks or more, with no previous history of treatment for TB, whose two sputum smears were acid-fast bacilli (AFB) negative in a baseline survey and were followed at community level [22]. Neighbourhood controls consists of 1,443 people who have no previous history of treatment for TB, and involved in the same study with the smear-negative patients with symptoms of TB [22]. Neighbourhood controls were selected from the first and fifth households to the right side of the houses of patients with TB suggestive symptoms. When we failed to select houses to the right, houses to the left were chosen. The rest of population includes 19,576 persons without history of treatment for TB, among whom TB cases were detected and reported by another study [6]. Figure 1 shows the study profile.

\subsection{Data Collection}

TB screening questionnaire was used to collect data from smear-negative patients with symptoms of TB and their neighbourhood controls. Every 3-4 months, enumerators interviewed the study subjects and asked if they have experiencing symptoms like cough for two weeks or more, blood in the sputum, fever, loss of appetite, weight loss, chest pain, shortness of breath, and night sweats. Sputum smear investigation was done as required. Patients brought sputum to health posts. HEWs collected sputum samples and then laboratory technicians prepared smears at health post, stained and read slides in the nearby health center. Between the surveys, smear-negative patients with persistent symptoms were advised to visit health facilities for more investigation [22]. Data from smear-negative patients with symptoms of $\mathrm{TB}$ and the neighbourhood controls collected during the census and subsequent surveys were registered.

In the latter TB case-finding approach, the HEWs collected sputum, prepared and sent smears to the nearby health center. Then laboratory technicians stained smears and did sputum microscopy in the health center. This was the 
main approach of TB case finding for the rest of population in the study area. While HEWs continued to do community based TB case finding, a few patients from this group went to health facilities for TB diagnosis through self-referral. About $12 \%$ of the TB cases were detected among self-referred patients.

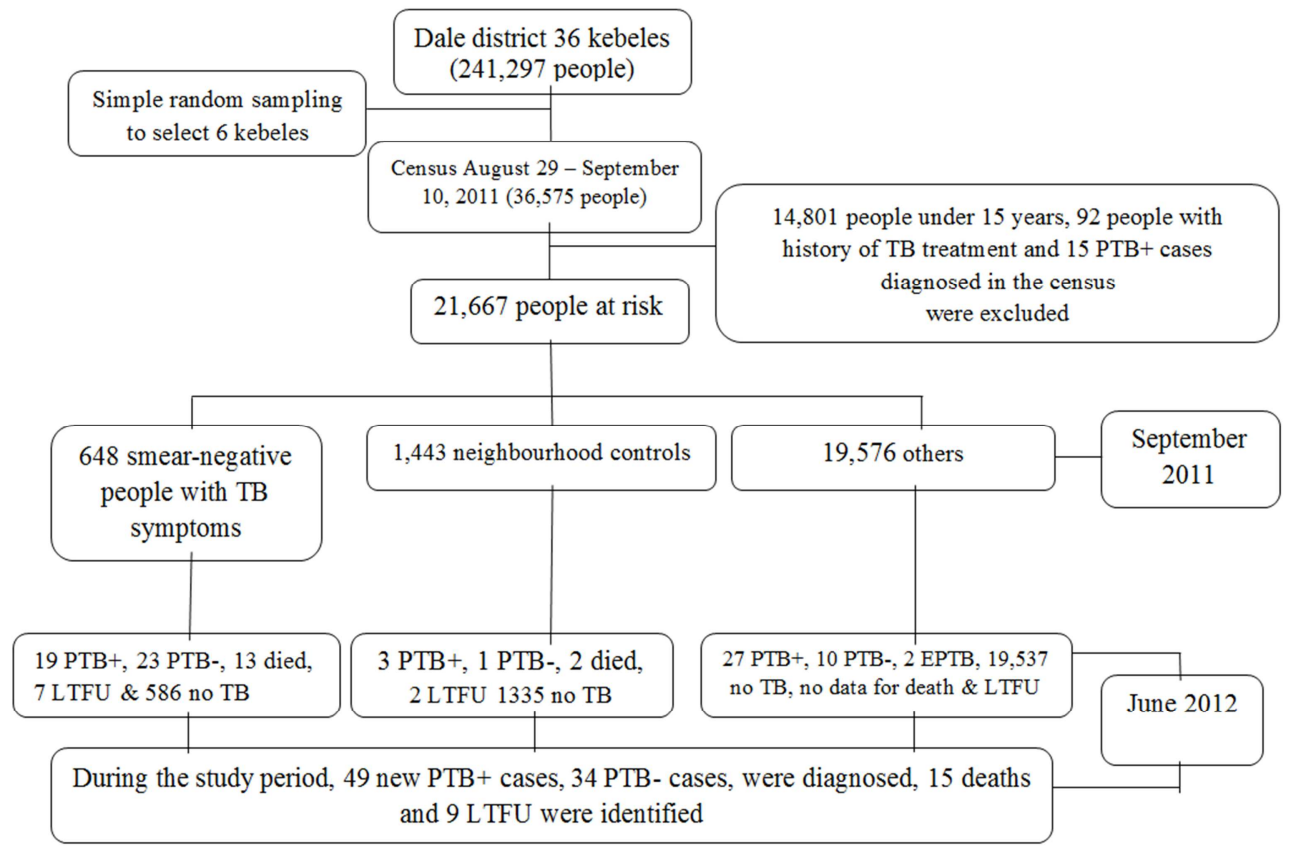

Figure 1. Cohort flow chart. Others $=$ the rest of population, $T B=$ tuberculosis, $L T F U=$ loss to follow-up, PTB $+=$ smear-positive TB, PTB- $=$ smear-negative $T B$.

Sputum microscopy was used to diagnose TB both in the census and in the follow-up surveys. Sputum microscopy was done twice on morning-spot basis, as there was evidence that microscopy can be optimized by examination of only two sputum specimens [23]. Patient with at least one AFBpositive sputum smear was diagnosed as a case of TB. Trained high school graduates who lived in the same communities with the study participants collected data.

\subsection{Quality Control and Data Processing}

The HEWs were trained on sputum collection, preparing and fixing smears at community level and slide storage. Laboratory technicians also received refresher training on staining and External Quality Assurance (EQA) procedures [6]. The TBREACH project delivered the trainings for both the HEWs and the laboratory technicians [6]. Based on the national guideline for the quality assurance of smear microscopy for TB diagnosis [24], EQA of smear microscopy was done for sample of slides [6, 24]. Discordant grades between the peripheral and the reference laboratories readings were below $1.6 \%[6,22]$.

The principal investigator (PI) delivered training to data clerks and supervised the data entry. During the study period, TB cases were registered and started their treatment at the nearby health center. Then patients were transferred to the health posts where the HEWs also register them and supervise the follow-up treatment. The health posts were located in their respective communities. Hence, TB patients from neighbouring communities or districts are unlikely to be included. All TB cases detected in the study communities were reported to the district health office and registered.

\subsection{Statistical Approach}

Data were entered using SPSS 20 statistical software. Analysis was done using SPSS version 20 statistical software. Smear-positive TB was the outcome variable, while gender, age, and education were background variables. Household size was considered as potential risk factor. The variables household size and age were re-coded into categorical variables using their median value, 6 people for household size and 35 years for age. Educational status was grouped in to "Illiterate" which consisted of people who have no schooling, while all other participants were grouped into "Literate" category.

Incidence rate of smear-positive TB was the the outcome variable determined by dividing new cases of smear-positive TB detected during the follow-up by person-years of observation. Person-time at risk of smear-positive TB was measured in years from the start date of follow-up until participants were diagnosed with smear-positive TB, died, lost follow-up, or end of the follow-up. The crude and adjusted IRR of smear-positive TB with the $95 \%$ confidence interval was reported.

\subsection{Ethics Statement and Consent to Participate}

The study protocol was approved by the Southern Nations Nationalities and Peoples' Regional (SNNPR) Health Bureau Research Ethics Review Committee in Ethiopia and the Regional Committees for Medical and Health Research Ethics 
(REK Nord) in Norway. Letter of support was obtained from the Sidama Zone Health Department. Informed verbal consent was obtained from the study participants. Smear-negative patients with symptoms of TB were treated with broad spectrum antibiotics and TB patients were treated with DOTS according to the National Guideline [22].

\section{Results}

\subsection{Cohort Characteristics}

A total of 21,667 adult people were followed for a total of 18,031 person-years of follow-up. Of this participants, $11,263(52.0 \%)$ were male and 12,680 (58.5\%) were illiterate. Nearly two-third of the study participants $(14,291)$ were below 35 years of age.

\subsection{Incidence and Risk Factors of Smear-Positive TB}

During the study period, 49 new smear-positive TB cases were identified. Incidence rate of smear-positive TB cases was 273 (95\% CI: 206 - 361)/100,000 person-years. Results of bivariate cox regression analyses showed that age (Crude IRR 2.0; 95\% CI 1.2 - 3.5) and education (Crude IRR 2.8; 95\% CI 1.4- 5.5) were associated with the risk of smear-positive TB. In a multivariate analysis however, only educational status maintained the significance in predicting smear-positive TB (Adjusted IRR $2.4 ; 95 \%$ CI $1.2-5.0)$ (see the Table 1$)$.

Table 1. Incidence rate and incidence rate ratio of smear-positive tuberculosis.

\begin{tabular}{|c|c|c|c|c|c|c|}
\hline Variables & TB cases & Persons involved & Person-years & IR/10 5 person-years & cIRR $(95 \%$ CI $)$ & aIRR (95\% CI) \\
\hline Incidence & 49 & 21,667 & 18,031 & $273(206-361)$ & & \\
\hline \multicolumn{7}{|l|}{ Gender } \\
\hline Male & 26 & 11,263 & 9,370 & $279(190-409)$ & & \\
\hline Female & 23 & 10,404 & 8,661 & $267(177-401)$ & $1.0(0.6-1.7)$ & $0.9(0.5-1.5)$ \\
\hline \multicolumn{7}{|c|}{ Age group in years } \\
\hline $15-34$ & 24 & 14,291 & 11,899 & $203(136-302)$ & & \\
\hline 35 & 25 & 7,376 & 6,132 & $409(277-506)$ & $2.0(1.2-3.5)$ & $1.6(0.9-2.9)$ \\
\hline \multicolumn{7}{|c|}{ Household size } \\
\hline 1 - 5 people & 27 & 10,184 & 8,475 & $320(219-466)$ & & \\
\hline 6 people & 22 & 11,483 & 9,556 & $231(152-351)$ & $0.7(0.4-1.3)$ & $0.8(0.4-1.4)$ \\
\hline \multicolumn{7}{|l|}{ Education } \\
\hline Illiterate & 39 & 12,680 & 10,547 & $371(271-508)$ & $2.8(1.4-5.5)$ & $2.4(1.2-5.0)$ \\
\hline Literate & 10 & 8,987 & 7,484 & $134(72-249)$ & & \\
\hline
\end{tabular}

$\mathrm{TB}=$ tuberculosis, $\mathrm{cIRR}=$ crude incidence rate ratio, aIRR $=$ adjusted incidence rate ratio, $\mathrm{IR} / 105$ person-years $=$ incidence rate per 100,000 person-years, $\mathrm{CI}$ $=$ confidence interval

\section{Discussion}

In this study, which consisted of both active and passive methods of data collection, a high incidence rate of smearpositive TB was found in rural settings of southern Ethiopia. Illiterate people had higher risk of smear-positive TB than literates.

The incidence of TB in this study was not different from the study report from northern Ethiopia [7]. They used a relatively similar design to the approaches we implemented [22]. It was a cohort study consisting of a three monthly survey to detect suspected cases of TB, at which time two sputum samples were collected for smear-microscopy [7]. Smear-negative TB suspects were treated with antibiotics and those without improvement were re-investigated for AFB. However, they measured incidence considering all smearpositive TB cases generated among adults aged 14 years [7] In the current study, only new smear-positive TB cases among people aged $>14$ years were considered.

Incidence of smear-positive $\mathrm{TB}$ in this study was similar to the 2012 WHO estimate for Ethiopia, 247/100,000 populations [4]. However, other studies reported high incidence of smear-positive TB than their respective national estimate $[7,25]$. The national estimate is for incidence of both new and recurrent TB cases, it is based on TB notification data, combined with expert opinion about cases diagnosed but not reported, or not diagnosed at all [3, 4]. In contrary, we did community based TB case finding supplemented by passive case finding for smear-positive TB cases, which probably increased the diagnosis of smearpositive TB by improving access to diagnosis.

In a population based cohort study in China, higher incidence rate of TB among men than women was reported for all age groups [10]. However, the WHO estimated maleto-female ratio of smear positive $\mathrm{TB}$ cases for the same country was 1:2.5 in 2012, showing no significant difference [4]. Authors of the study suggested difference in risks of infection and lifestyles may account for the gender difference on incidence of TB [10]. In the current study, the incidence rate of TB was similar for men and women. This was in agreement with the WHO estimation for Ethiopia in 2012, which was a 1:1.2 male-to-female ratio of incidence of smear-positive TB $[4,22]$. In contrary to this, other studies from Ethiopia, which used active case finding approach, reported higher incidence rate of smear-positive TB among women $[6,7,26]$. This was mainly due to active case finding approach minimized geographic and socioeconomic barriers to service access for women $[6,7,26]$. In passive case finding approach usually more men smear-positive TB cases are detected than women [27].

In Croatia and India, education was considered as one of the 
predictors of TB $[28,29]$. A similar finding was reported from northern Ethiopia [7]. In agreement to these reports, in the current study, illiterate people had over three fold higher risk of smear-positive TB than literate people. This could be related to illiterate individuals may have higher risk of disease transmission due to lack of knowledge on appropriate preventive measures of TB and are more exposed to TB infection.

Incidence of $\mathrm{TB}$ in this study may be over-estimated as some of the patients with symptoms of TB who were sputum smear-negative at the time of census would have been excluded as prevalent cases. Sputum culture and GeneXpert have higher sensitivity in detecting smear-positive TB than smear-microscopy $[30,31]$. If sputum culture or GeneXpert had been available and used or further sputum microscopy done for patients not responding to antibiotic treatment in the census, some of the patients with symptoms of TB identified with smear-positive $\mathrm{TB}$ in the cohort would have been excluded from the cohort. Similarly, during the follow-up there could be underestimation of the incidence rate due to not using the highly sensitive methods of TB diagnosis. However, similar diagnosis tool (sputum microscopy) was used both in the initial and follow-up surveys. In this study, using multiple methods of TB case finding might have introduced bias. Particularly the approach, case finding by HEWs was an open cohort study in which some people with TB suggestive symptoms might have been overlooked or unidentified and this may underestimate the incidence. Despite these limitations, the current study is one of the few population based studies which estimated incidence rate of smear-positive TB among adults in Ethiopia.

\section{Conclusion}

Incidence rate of smear-positive $\mathrm{TB}$ among rural population in southern Ethiopia was high. The disease is more common among illiterates. This shows that TB is still a major public health problem in rural communities of South Ethiopia. Maintaining the public health interventions being carried out in the study area is suggested. The author recommends that future studies should consider using sputum culture or GeneXpert in screening and diagnosis of TB cases. This could improve sensitivity and specificity of the base line screening and follow-up investigations.

\section{List of Abbreviations}

cIRR: crude incidence rate ratio; aIRR = adjusted incidence rate ratio; CI: confidence interval; DOTs: directly observed treatment short course, TB: tuberculosis, SPSS: statistical package for social science.

\section{Declarations}

\section{Ethics Statement and Consent to Participate}

The study protocol was approved by the Southern Nations Nationalities and Peoples' Regional (SNNPR) Health Bureau
Research Ethics Review Committee in Ethiopia and the Regional Committees for Medical and Health Research Ethics (REK Nord) in Norway. Letter of support was obtained from the Sidama Zone Health Department. Informed verbal consent was obtained from the study participants. Smear-negative patients with symptoms of TB were treated with broad spectrum antibiotics and TB patients were treated with DOTS according to the National Guideline. [22]

\section{Availability of Data and Materials}

The datasets used in this study are available from the corresponding author on reasonable request.

\section{Funding}

The study was financially supported by the University of Bergen. The funder had no role in designing the study and conducting the analysis.

\section{Author Contributions}

EMW conceived the study, analyzed and interpreted data and wrote the first draft manuscript. EMW approved the final manuscript.

\section{Author Details}

Hawassa University, College of Medicine and Health Science, School of Public Health, Hawassa, Ethiopia.

\section{Conflict of Interests}

none.

\section{Acknowledgements}

The investigator thanks data collectors, HEWs and the study participants. The author thanks Sidama Zone Health Department, REACH ETHIOPIA in Sidama, and Dale district health office. The author would also like to thank Prof Bernt Lindtjørn, Dr. Mohamed Ahmed Yasin and Dr. Daniel Gemechu for providing comments. Part of data was obtained from Dr. Mesay Hailu. The study was financially supported by the University of Bergen.

\section{References}

[1] WHO: Global tuberculosis report. In. Geneva, Switzerland: World Health Organization; 2014.

[2] Sharma R, Jain V, Singh S: Strengthening TB surveillance system in India: Way forward for improving estimates of TB incidence. Lung India 2011, 28 (2): 120-123.

[3] WHO: Global tuberculosis report. In. Geneva, Switzerlnd: WHO; 2015.

[4] WHO: Global tuberculosis report. In. Geneva, Switzerland: World Health Organization; 2013. 
[5] Shargie EB, Mørkve O, Lindtjørn B: Tuberculosis casefinding through a village outreach programme in a rural setting in southern Ethiopia: community randomized trial. Bulletin of the World Health Organization 2006, 84: 112-119.

[6] Yassin MA, Datiko DG, Tulloch O, Markos P, Aschalew M, Shargie EB, at et: Innovative community-based approaches doubled tuberculosis case notification and improve treatment outcome in southern Ethiopia. PLoS One 2013, 8 (5): 63174.

[7] Tadesse T, Demissie M, Berhane Y, Kebede Y, Abebe M: Incidence of smear-positive tuberculosis in Dabat, northern Ethiopia. Int J Tuberc Lung Dis 2013, 17 (5): 630-635.

[8] Chen W, Shu W, Wang M, Hou Y, Xia Y, Xu W, al et: Pulmonary tuberculosis incidence and risk factors in rural areas of China: A cohort study. PLoS One 2013, 8 (3): e58171.

[9] Van LF, Vander WMJ, Borgdorff MW: Prevalence of tuberculosis infection and incidence of tuberculosis: a reassessment of the Styblo rule. Bull World Health Organization 2008, 86: 20-26.

[10] Wondimu T, W/Michael K, Kassahun W, Getachew S: Delay in initiating tuberculosis treatment and factors associated among pulmonary tuberculosis patients in East Wollega, Western Ethiopia. Ethiop J Health Dev 2007, 21 (2): 148-156.

[11] Yimer S, Holm CH, Yimaledu T, Bjune G: Health care seeking among pulmonary tuberculosis suspects and patients in rural Ethiopia: a community-based study. BMC Public Health 2009, 9.

[12] Demisse M, Lindtjorn B, Berhane Y: Patient and health service delay in the diagnosis of pulmonary tuberculosis in Ethiopia. BMC Public Health 2002, 2.

[13] Dangisso MH, Datiko DG, Lindtjørn B: Accessibility to tuberculosis control services and tuberculosis programme performance in southern Ethiopia. Glob Health Action 2015, 8

[14] Dye C, Watt CJ, Bleed DM, Hosseini SM, Raviglione MC: Evolution of tuberculosis control and prospects for reducing tuberculosis incidence, prevalence and deaths globally. JAMA 2005, 293 (22): 2767-2775.

[15] FMOH: Federal Ministry of Health of Ethiopia. TB control programme annual performance report 2010/11. In. Addis Ababa, Ethiopia: Federal Ministry of Health of Ethiopia; 2011.

[16] FMOH: Health extension programme implementation guidelines. In. Addis Ababa, Ethiopia: Federal Ministry of Health Ethiopia; 2007.

[17] Negusse H, Auliffe EM, Maclachlan M: Initial community perspectives on the health service extension programme in Welkait, Ethiopia. Hum Resour Health 2007, 5.

[18] FMOH: Health and health related indicators. In. Addis Ababa, Ethiopia: Ministry of Health; 2011.
[19] Kebede AH, Alebachew Z, Tsegaye F, Lemma E, Abebe A, Agonafir M, al et: The first population-based national tuberculosis prevalence survey in Ethiopia. Int J Tuberc Lung Dis 2004, 18 (6): 635-639.

[20] WHO: Global tuberculosis report. Annex 2: Country Profile, Ethiopia. In. Geneva, Switzerland: World Health Organization; 2012.

[21] Yassin MA, Datiko DG, Shargie EB: Ten-year experiences of the tuberculosis control programme in the southern region of Ethiopia. Int J Tuberc Lung Dis 2006, 10: 1166-1171.

[22] Woldesemayat EM, Datiko DG, Lindtjørn B: Follow-up of chronic coughers improves tuberculosis case finding: Results from a community based cohort study in southern Ethiopia. PLoS One 2015, 10 (2): e0116324.

[23] Ramsay ARC: Making the most of poor diagnostics: increasing access to tuberculosis treatment through optimized smear microscopy services. In: In: Steingart KR, Ramsay A, Pai $M$, eds Optimizing sputum smear microscopy for the diagnosis of pulmonary tuberculosis Expert reviews in antiinfective therapy. Volume 5, edn.; 2007: 327-331.

[24] EHNRI: Guidelines for quality assurance of smear microscopy for tuberculosis diagnosis. In. Addis Ababa, Ethiopia: Ethiopian Health and Nutrition Research Institute; 2009.

[25] Pronyk PM, Kahn K, Tollman SM: Using health and demographic surveillance to understand the burden of disease in populations: the case of tuberculosis in rural South Africa. Scand J Public Health 2007, 35: 45-51.

[26] Datiko DG, Lindtjørn B: Health extension workers improve tuberculosis case detection and treatment success in southern Ethiopia: A community randomized Trial. PLoS One 2009, 4 (5): e5443.

[27] Connolly M, Nunn P: Women and tuberculosis. World health stat $Q$ 1996, 49: 115-119.

[28] Savicevic AJ, Mulic R, Ban B, Kozul K, Ivcek LJ, Valic J: Risk factors for pulmonary tuberculosis in Croatia: A matched case-control study. BMC Public Health 2013, 13: 991.

[29] Shetty N, Shemko M, Vaz M, D'Souza G: An epidemiological evaluation of risk factors for tuberculosis in South India: a matched case control study. Int J Tuberc Lung Dis 2006, 10 (1): 80-86.

[30] AmericanThoracicSociety: Diagnostic standards and classification of tuberculosis. Am Rev Respir Dis 1990, 142 (3): 725-735.

[31] Laushkina Z, Cherednichenko A: Estimation of the role of the test GeneXpert MTB-RIF in the diagnosis of pulmonary tuberculosis. ERJ 2014, Suppl 58 (44): 2677. 